\title{
Three-dimensional Digital Realization of Cultural Heritage
}

\author{
Hongfu He \\ Weinan Normal University, Weinan, Shaanxi, 714099
}

Keywords: Cultural heritage; three-dimensional digitization; model

\begin{abstract}
Cultural heritage is the historical legacy of human civilization. It embodies the ancient and splendid civilization tradition. It is a kind of cultural blood, inheriting history and showing the future. Today, with the development of the digital cultural industry, the use of three-dimensional digital technology to collect, store, process and display precious cultural heritage makes the historical remains of human beings closely related to people's daily lives, and provides more advanced implementation solutions for the inheritance and innovation of China's excellent traditional culture.
\end{abstract}

\section{Introduction}

Article 1 of the United Nations Educational, Scientific and Cultural Organization (UNESCO) Convention for the Protection of the World Cultural Heritage [1] (1972) defines cultural heritage as "cultural relics, buildings and sites". 2015 "Guidelines for the Protection of Chinese Cultural Relics and Monuments" [2] "Classification proposed the basic norms for the protection of new cultural heritage, and initially established a system of protection criteria covering various types of cultural heritage and relatively complete Chinese cultural heritage." Regulations: Cultural relics and monuments refer to valuable immovable physical remains created or left by human beings in history, including ancient cultural sites, ancient tombs, ancient buildings, cave temples, stone carvings, modern and historical sites and representative buildings, historical culture. Famous cities, famous towns, famous villages and their affiliated cultural relics; cultural heritage, cultural routes, heritage canals and other types of heritage are also within the scope of cultural relics."

The "Cultural Heritage" in the Convention for the Protection of the World Cultural Heritage and the "Cultural Heritage Monument" in the "China Guidelines" are a set of concepts that are very similar in meaning, including cultural relics, architectural complexes, and sites. Some scholars use and distinguish between "cultural heritage" and "cultural heritage" according to the regulations. In fact, even if there are some differences between the two, it will be very small.

Image processing technology, 3D modeling technology, laser scanning technology, virtual display technology and data preservation technology continue to develop, and it has become possible to store, display and transform cultural heritage in a three-dimensional digital way. Creating a three-dimensional model and texture of cultural heritage in a harmless, omni-directional manner, optimized and integrated into a resource that can be used not only to extend the limits of cultural heritage protection and restoration, but also to apply to digital cultural creativity, digital creativity and related The key direction of digital creative industries such as industrial integration development and design services is to realize resource sharing, expand display space, and use 5G technology to promote the next generation of digital entrepreneurial art works, and promote virtual reality and secondary culture to be rooted in Chinese culture.

The basis of three-dimensional digitization of cultural heritage is the three-dimensional model. The creation of 3D models requires a variety of software support, including 3ds Max (Max) for building modeling, Maya; SpeedTree (hereinafter referred to as ST), a specialized software for creating tree models; and specialized software for creating natural landscapes. VuexStream (hereinafter referred to as VUE), lumion; image processing software Adobe Photoshop (hereinafter referred to as PS), texture processing software Quixel Suite, Substance Painter kit (hereinafter referred to as SP); digital engraving and painting software ZBrush (hereinafter referred to as ZB), Mudbox. 
The 3D model is one of the foundations and key elements of the digitalization of cultural heritage. According to the purpose of the 3D model, it can be divided into a low polygon model and a high polygon model. In general, low-polygon models are used in three-dimensional games, virtual reality design, archaeology, and the like. Can be subdivided into "online specifications" and "secondary specifications" model [4]. Online game specifications generally use low-surface model, texture less than $1024 * 1024$ pixels and less texture pass, they are often used in the development of online games and mobile games. The next-generation specifications generally use medium-faceted models, relatively large textures, and more texture channels, which are often used in game development such as high-performance PCs and mainframes. High polygon models can be used in 3D animated movies, game cutscenes, and promos. In the film, the model can be divided into three fine types: low, medium and high. Compared with the model needed for real-time rendering, the model of the film specification does not have many strict rules, but has more freedom and broad space.

Textures are an important part of improving the look and feel of 3D models. PBR technology, which has been widely used abroad, is an abbreviation of English Physical-Based Rendering, which means a rendering technique based on physical law simulation. This technology was first used in the photo-real rendering of movies, and has been widely used in real-time rendering of PC games and console games in recent years. The technology mainly has intrinsic color map, normal map, roughness map, metal texture map (or gloss map), ambient light occlusion map, detail map and so on.

\section{The technical route of three-dimensional digitalization of cultural heritage}

The general idea of the three-dimensional digital practice of cultural heritage is: after obtaining the first-hand materials of cultural relics, architectural complexes, cultural landscapes and other heritages through personal art collection, draw the vitality of the artistic origin, learn the multi-level cultural connotation of art, and infiltrate it into three-dimensional Model creation, texture mapping processing, rendering output, etc., and the practical results as a resource for animation, film, virtual reality and other fields.

The three-dimensional digital practice of cultural heritage is gradually carried out according to the method and technical route shown in Figure 1. The focus is on the collection of original materials; the classification and digitization of materials; the three-dimensional model creation techniques of ancient buildings, the models of ancient and famous trees and animation techniques, the three-dimensional model creation techniques of cultural relics; the application of three-dimensional model resources in roaming and virtual reality Wait.

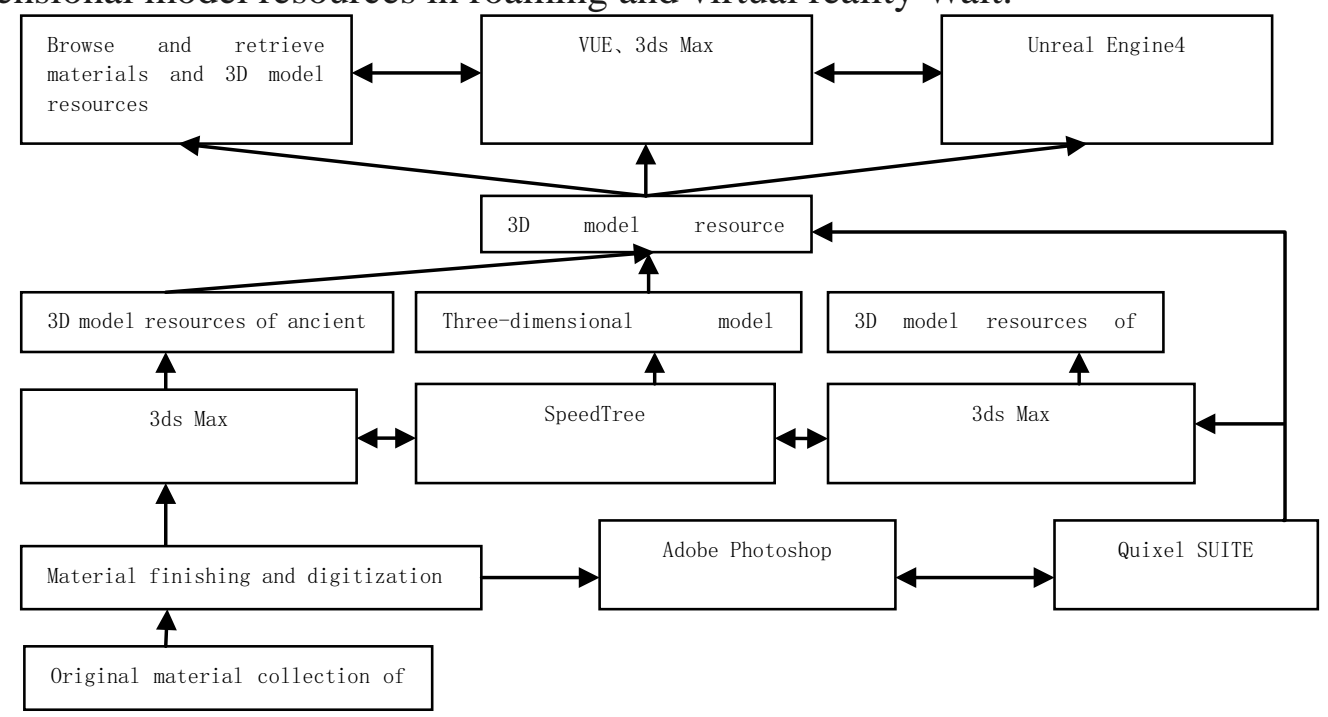

Fig 1 Technical roadmap for 3D digital realization of cultural heritage 


\section{The main links of three-dimensional digitalization of cultural heritage}

Cultural heritage art collection is the starting point of 3D model creation. The methods of collecting wind mainly include: using human measurement method, organizing manpower site measurement; collecting data by interview method; using sketching, sketching, color and digital photography to record images, record images data.

Sketching is an important method of artistic collection. Some people say that when the camera shutter is pressed, all the images that are included in the lens are recorded. What else is drawn? Facts have proved that the relationship between photography and painting is very clear. Photography can be recorded, painting can not be done; painting can do, photography does not necessarily do. Although there is a connection between the two, they cannot be replaced. Painting pays attention to the inner vision and inner lens of the creative subject, and the subjective activity scale is larger, especially the modulation of "scenario" is almost "creation" [5].

The purpose of sketching is not just to collect material for creation. Because the creator, in addition to improving the necessary styling ability and aesthetic ability, must also improve the ability to observe, express, and create images through memory and imagination through sketching. Moreover, in the face of the same heritage sketch, each author's work should be different. Therefore, sketching helps creators to "discover" and "distort". "Discovery" is what is found in cultural heritage. "Difference" is different from prototype and different from others in artistic creation. Artists do not have a style without paying attention to personality, and they do not call it art without paying attention to style.

Using a digital camera to capture the overall and local details of the omnidirectional, multi-view, detailing the color and texture information on the cultural heritage is very helpful in creating realistic 3D models. However, it is not a matter of pressing the shutter. First of all, because the height of the person is shorter than that of the building, the photograph is often viewed from the angle of view. Therefore, the object on the photo has a large perspective angle. Secondly, it is difficult to obtain a full view from a narrow street or courtyard. The image of the main facade, side and top of the ancient building; and the debris such as trees, telephone poles and grasshoppers will cover some of the buildings, so that the color and texture information obtained is not complete enough; although the creators carefully capture each In detail, it is difficult to avoid discovering that some content is still missing after returning to production, so the creator should pre-set the collection plan before the departure to avoid unnecessary trouble. Despite this difficulty, the art collection work still has to adhere to the principles of science and authenticity, the structural form of the components in a building, the way the stone is laid on a road, and even the font, color and texture of a piece of plaque need to be collected one by one.

Field trips and live experiences are essential to deepen the credibility and presence of digital works. The pictures of some ancient buildings, although they can be found in the literature, are not a substitute for the rare personal experience of the creators. Experience the breath, sound and light, and buy some local specialties, hope to become a real props. Without field trips, it is difficult for people to understand what a cypress tree of more than 5,000 years old looks like! This is a feeling that it is difficult to convey the second-hand information such as language descriptions, pictures, videos, etc., and, in the landscape of "has never seen such a large, ancient tree", the feeling of blending scenes cannot be copied. The creative practice proves that only by incorporating these elements of personal experience into the work will it bring a real feeling of affection to the audience. This is the core role of art collection.

Through the 3D laser scanner, the model and texture information of the cultural relics are accurately and efficiently recorded in a non-contact, non-damaging, all-round digital manner.

Obtain topographic maps, contour maps, and elevation data through GIS to provide basic data for the construction of related cultural heritage scenes. Collect archaeological data on relevant cultural heritage and organize the data needed to create the model.

The method of creating terrain with reference to geographic information can maximize the original terrain. There are many ways to obtain geographic information. It is an efficient way to obtain topographic maps, contour maps, and elevation data through GIS. For example, use the 
BiGEMAP map downloader.

The creation of 3D model resources is a complex project that requires a scientific workflow. The process is not only to consider the problem of improving efficiency or saving investment from the perspective of fault tolerance (prevention and response to errors in the work), but more importantly, how to improve the quality of the work from the perspective of creation.

The workflow for 3D model resource creation includes project planning, material sorting and digital image processing, 3D model creation, texture processing, and quality monitoring throughout.

Project planning

In order to complete the task of planning the project, detailed standards and requirements are required. Work plans such as schedules, collection plans, processing standards, model specifications, and output formats are developed to ensure that all aspects of data collection, model making, and mapping are progressed in an orderly and timely manner to reduce or avoid unnecessary problems.

The images collected are scattered and the number is huge. In order to satisfy the convenient application of images, the images should be classified and managed according to the hierarchical method of the project. The image file hierarchy can be stored in accordance with the principle of "project name - cultural attribute - spatial position - angle number".

Factors such as multiple shooting angles, changing lighting environments, different times, and being obscured by other objects cause the hue, brightness, and purity of the original image material to be collected, and the shape and structure are deformed. Process it. Working group personnel use Adobe Photoshop image processing software (because the software is very popular software, this book will not introduce the basic operation and editing skills of the software.), the image processing. The main aspects of processing include image noise reduction, perspective correction, image stitching, sharpness adjustment, uniform resolution, image segmentation, etc. to meet the requirements of modeling and texture design and production.

In the field of animation, games, and digital media art, three-dimensional models created with reference to cultural heritage existing in the real world can be classified into scene model classes. The scene model is proposed by the relative role model. It generally includes terrain, architecture, roads, sculptures, trees, and other types of models. They usually do not involve deformation animation (but trees can involve wind animation, growth animation).

The main means of data quality monitoring is to conduct data review, the purpose of which is to determine whether each process meets the requirements of project planning. The basis for the decision is the schedule, the acquisition plan, the processing criteria, the model specifications, and the output format. By finding the wrong problems of modeling and mapping, the rework modification is carried out to ensure the completeness and accuracy of the model data, so that the 3D model resources designed and manufactured with reference to the cultural heritage can satisfy the film production, the design and development of cultural relics derivative products, and the virtual reality display. Actual demand.

The way of 3D model resource review is mainly the combination of internal and external review. Internal review is a mutual inspection between members, mainly to solve obvious mistakes; external review is to invite industry experts to review and help find professional problems.

If the three-dimensional model of cultural heritage is used as a resource, it should be able to play a greater role in more fields. However, different fields, such as animation, games, virtual reality, etc., have different requirements for 3D model resources; different specifications of the same application domain, such as game game online game specifications, sub-era specifications, PBR framework, etc., accuracy of 3D model resources There are also some differences in texture requirements, and even different platforms, such as virtual reality Unity, Unreal, have different requirements for texture materials for 3D models. Therefore, it is necessary to integrate the basic resources of the 3D model to meet the requirements of another application field.

Model files with clear classification, model optimization, and production specifications are the basic requirements for $3 \mathrm{D}$ model resource integration. When a $3 \mathrm{D}$ model resource applied to a movie is created, the scene unit size, the model and texture naming, the model's axis coordinates, texture format and format, model surface normals, animations, etc. must conform to the film's 
requirements. If you want to apply 3D model resources to movies and apply them to virtual reality projects, you must integrate existing resources according to the requirements of virtual reality for 3D model resources. On the basis of the model resources applied to a domain, after model re-optimization, texture re-processing, and file reclassification, it is suitable for application in another field, which is conducive to improving work efficiency or saving investment, and is more conducive to improvement. The quality of the work.

\section{Conclusion}

As far as the three-dimensional digitization of cultural heritage is concerned, the application of technology is very mature, but the key link is still the requirement of three-dimensional model resources for different purposes, that is, the standardization of the fineness of the model, as well as the size and type of the texture and the actual color and texture of the object. Management of the degree of reproduction or performance. From a practical perspective, team members' synergy, technical ability and artistic literacy all affect the quality of three-dimensional digitalization of cultural heritage.

\section{Acknowledgements}

Fund Project: This paper is the research result of the key research project of Weinan Normal University, "Research and Practice of First-class Professional Construction of Animation" (Project No.: 302317013).

\section{References}

[1] Convention for the Protection of World Cultural and Natural Heritage [A]. Chinese Ethnological Society. National Culture and Globalization Seminar Information Album [C]. Chinese Ethnological Society, 2003: 10.

[2] International Committee of Monuments and Sites, China National Committee, 2015. Guidelines for the Protection of Chinese Cultural Relics and Monuments [M]. Beijing: Cultural Relics Publishing House, 2015.

[3] Yang Gang, Luo Weiliang. Technology-Art Thinking [M]. Xi'an: Northwest University Press, 2010.

[4] Liu Xiangqun waits, VR/AR/MR development combat [M]. Beijing: Mechanical Industry Press, 2017: 47.

[5] Xie Chunyan. Ye Shaoyu talks sketch [M]. Shanghai: Shanghai People's Fine Arts Publishing House. 2007: 16.s 